\title{
Comparative Review on Efficient Design of Reversible Sequential Circuits based on Optimization Parameters
}

\author{
Gobinda Karmakar ${ }^{1}$, Dr. Saroj Kumar Biswas ${ }^{2}$, Dr. Ardhendu Mandal ${ }^{3}$, Arijit \\ Bhattacharya ${ }^{4, *}$, Akhil Kumar Das ${ }^{4}$, Ekram Alam ${ }^{4}$ \\ ${ }^{1}$ Department of Computer Science, Acharya Prafulla Chandra College,New Barrackpore, \\ West Bengal 700131 \\ ${ }^{2}$ Department of Computer Science \& Engineering, NIT Silchar, India \\ ${ }^{3}$ Department of Computer Science \& Application, University of North Bengal, West \\ Bengal, India \\ ${ }^{4}$ Department of Computer Science, Gour Mahavidyalaya, Malda, West Bengal \\ 732142, India
}

\begin{abstract}
Reversible computing, a well known research area in the field of computer science. One of the aims of reversible computing is to design low power digital circuits that dissipates no energy to heat. The main challenge of designing reversible circuits is to optimize the parameters which make the design costly. In this paper, we review different designs of efficient reversible sequential circuits and prepare a comparative statement based on eight optimization parameters such as Quantum Cost (QC), Delay (del), Garbage Output (GO), Constant Input (CI), Gate Level (GL), Number of Gate (NoG), Type of Gate (ToG), Hardware Complexity (HC) of Circuit.
\end{abstract}

Keywords: Reversible Computing, Sequential circuit, optimization parameters, low

\section{INTRODUCTION}

Computers play an important role in efficient information processing as society's productivity and economy grow. Technological advancement in the computation field continues to improve as fast as possible for our social interests. It is progressed through the improvement in the speed and power of computers. In recent year Reversible computation is considered an important way to improve the use of power of computers. Logical reversibility is the logical state of the computational device just prior to the operation (its input state) which is uniquely determined by its state just after the operation (its output state) and physical reversibility is a process that dissipates no energy to heat, and produces no entropy [1]. It is proven that each bit of information loss generates kTln2 joules of heat energy, where k is Boltzmann's constant and $\mathrm{T}$ is the absolute temperature at which computation is performed [2]. In reversible computation, information loss not has done during computation process due to its one to one correspondence between its inputs and outputs. It is shown that the circuit with reversible logic gates can avoid the heat dissipation [3]. The optimization of the reversible circuit is the main design issue based on different parameters which result the design costly. The most important parameters are Quantum Cost (QC), Delay (del), Garbage Output (GO), Constant Input (CI), Gate Level (GL), Number of Gate (NoG), Type of Gate (ToG), Hardware Complexity (HC) of Circuit. In this paper we review the existing works in Reversible Sequential Circuits and prepare a comparative statement based on different optimization parameters. Many researchers are involved to design new reversible sequential circuit. This paper helps them to measure the cost of their circuit and compare with other design to find the optimization of their circuit. The paper is arranged as follows: section 2 provides the description of Reversible sequential circuit and its design issues. Section 3 provides the description about the optimization parameters. Section 4 presents the reversible logic gates with its cost those are used to design sequential circuits. Section 5 presents the comparative study about different design of sequential circuits and finally this paper is concluded with section 6. 


\section{Design Issues of Reversible Sequential Circuit}

A sequential circuit consists of a combinational circuit to which memory elements are connected to form a feedback path. It receives binary information from external inputs, combine with the present state of memory elements and determine the binary value at the output terminals. That means a sequential circuit is specified by a time sequence of inputs, outputs and internal states. The external outputs in a sequential circuit are a function of external input and present state of memory element. A reversible sequential circuit is reversible if its transition function is constructed by reversible logic [4]. The transition function acts as a combinational reversible circuit [4]. The functionality of combinational reversible circuit is implemented by suitable reversible gate.

One of the issue that sequential circuit requires a feedback from one of the previous outputs but reversible logic gate does not allow a fan out from one signal [5]. For this 2-bit Toffoli gate is used to make duplicate signal [4]. Optimized design of reversible sequential circuit is another issue.

\section{Optimization Parameters}

In this paper, we have considered eight optimization parameters forevaluation and comparing among different design of reversible sequential circuits. From the survey of reversible circuits, it is observed that all eight optimization parameters are equally important to synthesis and it plays a pivotal role for the design of optimized reversible circuits.

3.1 Number of Gate (NG): Total number of gates used in circuit. It is proportional to circuit complexity. Minimum possible number of gates makes the circuit efficient.

3.2 Quantum Cost (QC): Total number of quantum gates used in circuit. It is very difficult to count the number of quantum gate of a circuit. So, the quantum cost of all 1x1 and $2 \times 2$ reversible gates are considered as unity [7]. Using the quantum constant of this unity we calculate the quantum cost of other circuit.

3.3 Delay (Del): The delay of a circuit is the maximum number of gates in a path from any input line to any output line [6]. In most cases delay has been calculate by the quantum cost.

3.4 Constant Input (CI): It is also known as Ancilla Input. The number of constant value (either 0 or 1) gives as an input to synthesize the logical function. Minimize possible constant input makes the circuit more generalized.

3.5 Garbage Output (GO): Unwanted output in circuit is called garbage output. Its present violate the reversibility of the circuits. Minimum number of garbage output makes the high performing reversible circuit.

3.6 Gate Level (GL): The number of level in the circuits. The level indicates that the output of one gate acts as a input of other gates. Its are required to realize the given logical function [6]. Minimization of gate level reduces the complexity of circuits.

3.7 Hardware : Complexity (HC): Its refer the number of AND, NOT and EX-OR operation present in circuit. It is shown that these three operations are functionally complete. That means using these operation we can implement any logical functionality. Minimization of these operations reduces the circuit complexity and cost. In this paper we consider $\alpha$ as a two input EX-OR operation, $\beta$ as a two input AND operation and $\gamma$ as a NOT operation.

3.8 Type of Gate (TG): The number of different type of gates is used in the circuit. Maximization of type of gate is responsible for increase of manufacturing complexity of circuit.

\section{Reversible Gate}

It is observed that the optimization parameters have not been considered properly in many proposed reversible circuits while designing. In this paper we calculate the quantum cost (QC) and Hardware Complexity (HC) with their reversible representation of the following reversible gates which is not mentioned in the respective paper.

4.1 MG-1 gate [8]: It is a $4 \times 4$ reversible gate. The block diagram with its functionality and reversible implementation is given in Fig. 1. The quantum cost of MG-1 gate is 8 . The hardware complexity is $5 \alpha+6 \beta+4 \gamma$. 

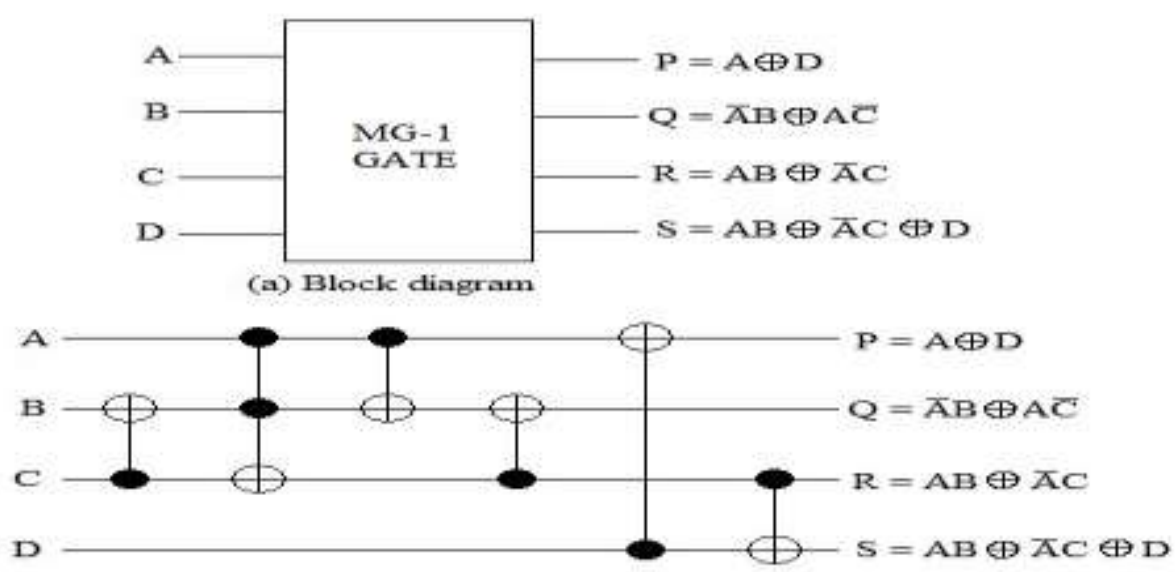

(b) Reversible Implementation

Fig 1: MG-1 gate and its reversible implementation

4.2 MG-2 gate [8]: It is a $4 \times 4$ reversible gate. The block diagram with its functionality and reversible implementation is given in Fig. 2. The quantum cost of MG-2 gate is 7. The hardware complexity is $3 \alpha+4 \beta+3 \gamma$.
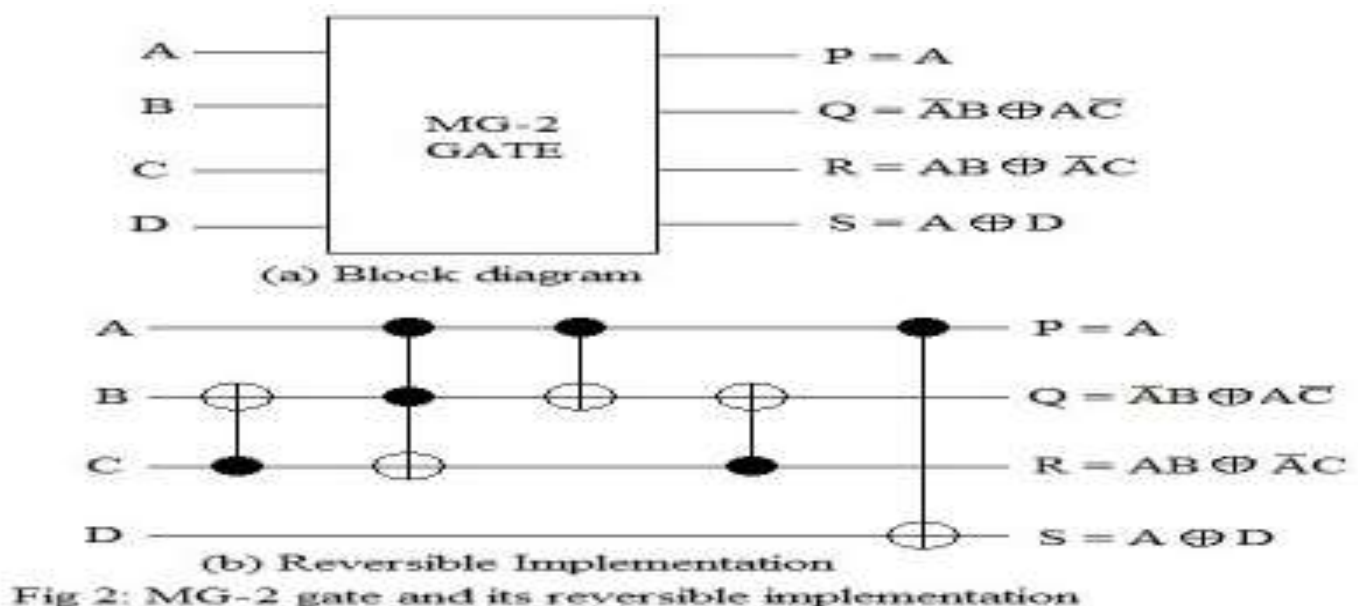

FiE $2:$ MCG-2 eate end its reversible implementation

4.3 SG gate [6]: It is a $4 \times 4$ reversible gate. The block diagram with its functionality and reversible implementation is given in Fig. 3. The quantum cost of SG gate is 9. The hardware complexity is $5 \alpha+6 \beta+3 \gamma$.

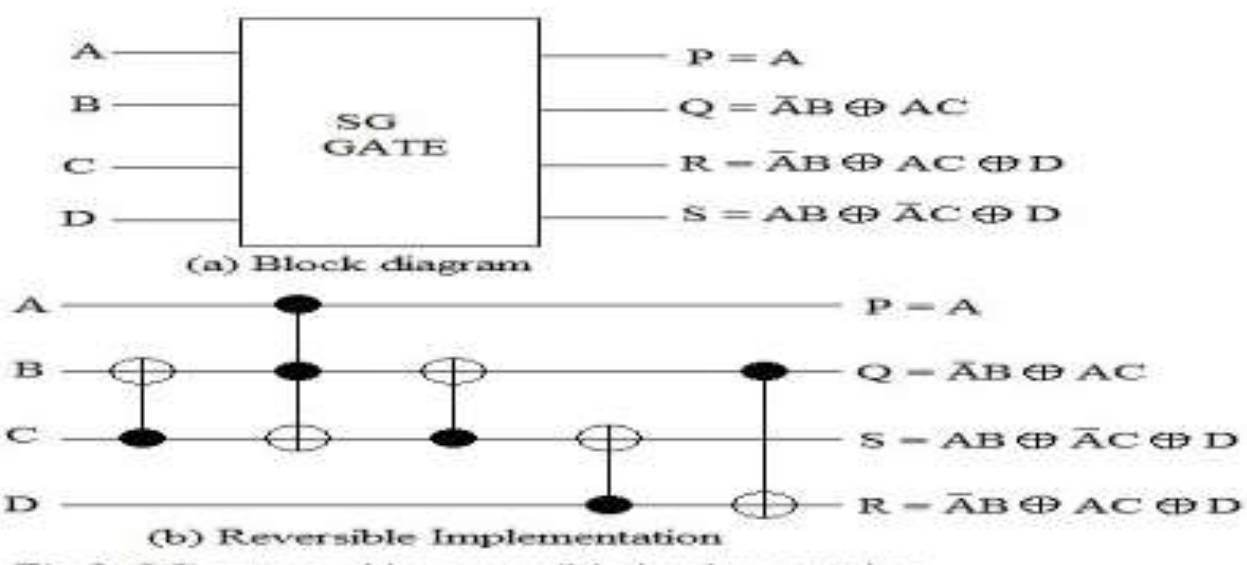

Fig 3: $S C$ g gate end its reversible implementation

4.4. NG gate [10]: It is a $3 \times 3$ reversible gate. The block diagram with its functionality and reversible implementation is given in Fig. 4. The quantum cost of NG gate is 11 . The hardware complexity is $2 \alpha+2 \beta+3 \gamma$. 

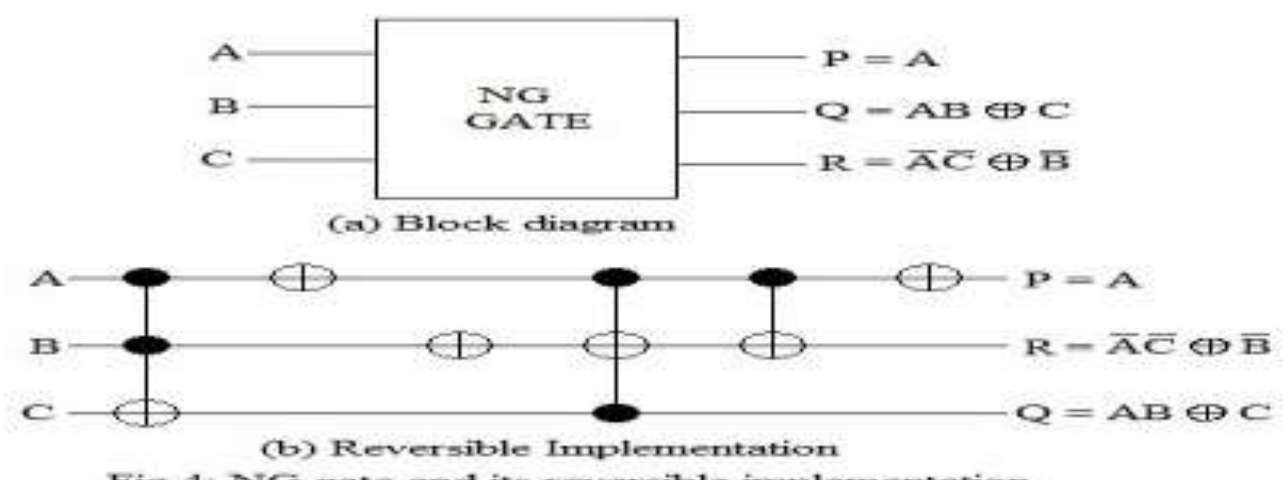

Fig 4: NG Eate and its reversible implementation

4.5 BME gate [11]: It is a $4 \times 4$ reversible gate. The block diagram with its functionality and reversible implementation is given in Fig. 5. The quantum cost of BME gate is 15 . The hardware complexity is $4 \alpha+3 \beta+\gamma$.

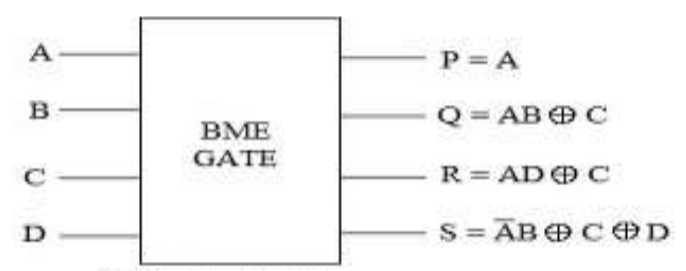

(a) Block diagram

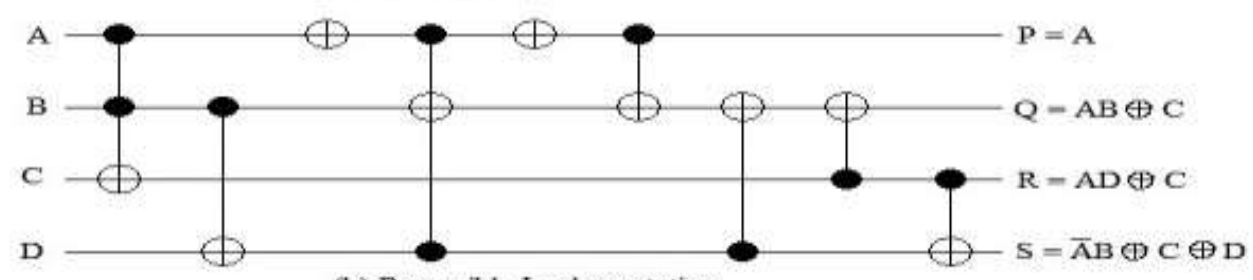

(b) Reversible Implementation

Fig 5: BME gate and its reversible implementation

4.6 URG gate [5]: It is a $3 \times 3$ reversible gate. The block diagram with its functionality and reversible implementation is given in Fig. 6 . The quantum cost of URG gate is 7 . The hardware complexity is $4 \alpha+2 \beta$.

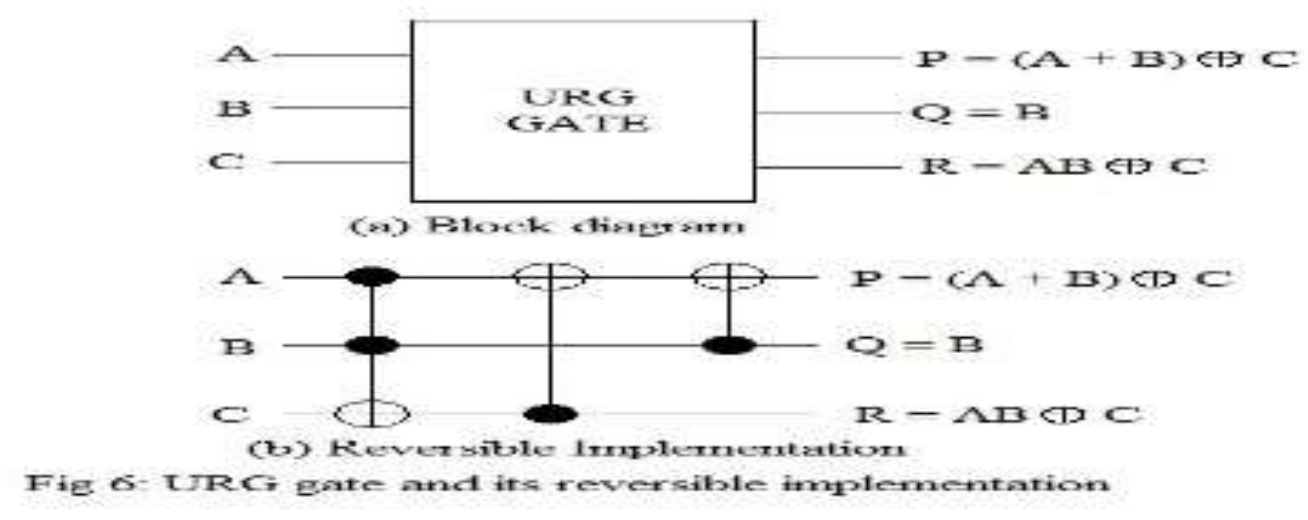

4.7 VB-1 gate[12]: It is a $4 x 4$ reversible gate. The block diagram with its functionality and reversible implementation is given in Fig. 7. The quantum cost of VB-1 gate is 11 . The hardware complexity is $4 \alpha+5 \beta+4 \gamma$. 

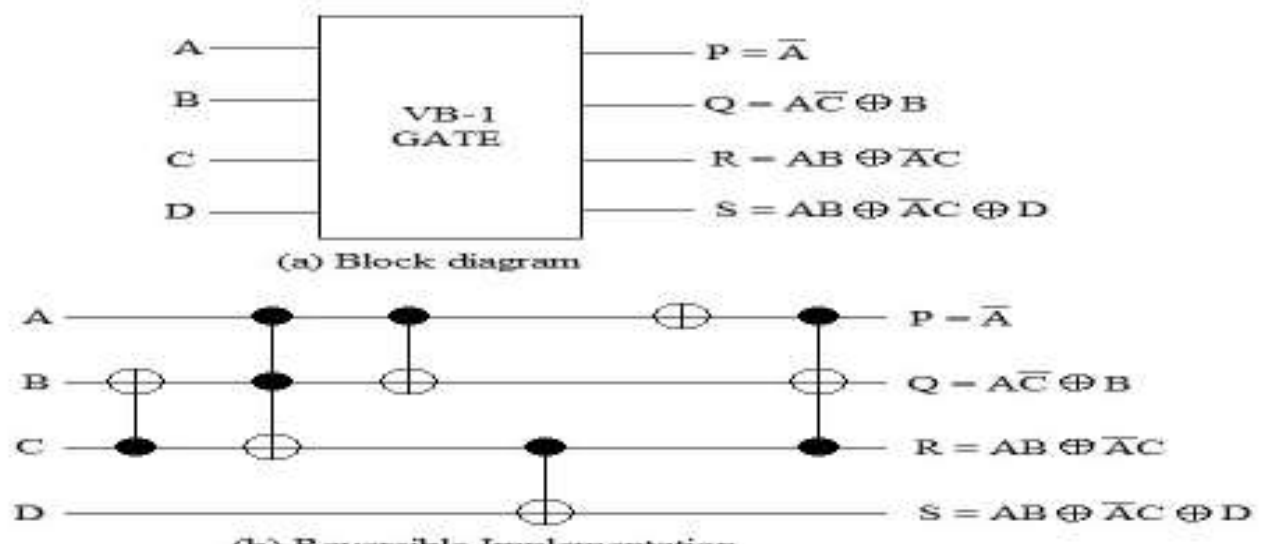

(b) Feversible Implementation

Fig 7: VB-I gate and its reversible implementation

4.8 VB-2 gate[12]: It is a $4 \times 4$ reversible gate. The block diagram with its functionality and reversible implementation is given in Fig. 8. The quantum cost of VB-2 gate is 8 . The hardware complexity is $3 \alpha+4 \beta+4 \gamma$.

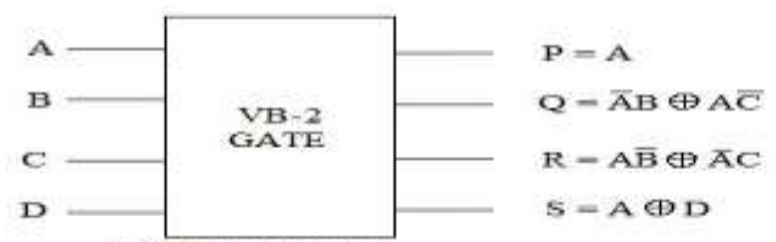

(a) Block diagram

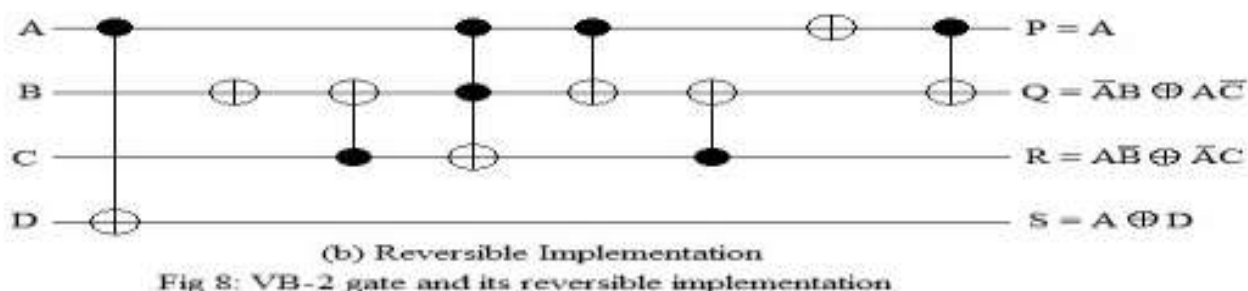

4.9 RSJ gate [14]: It is a $4 \times 4$ reversible gate. The block diagram with its functionality and reversible implementation is given in Fig. 9. The quantum cost of RSJ gate is 10. The hardware complexity is $2 \alpha+2 \beta$.

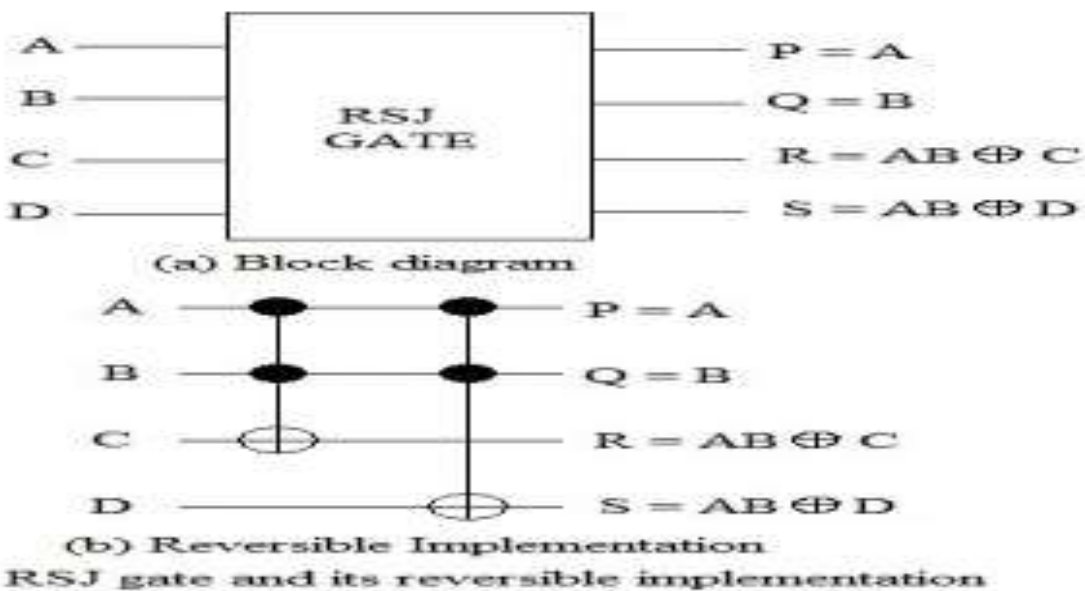

Fie $S$ : FSS Eate and its reversible implementeation

4.10 Mux gate [19]: It is a $3 \times 3$ reversible gate. The block diagram with its functionality and reversible implementation is given in Fig. 10. The quantum cost of MUX gate is 5. The hardware complexity is $3 \alpha+2 \beta+\gamma$. 


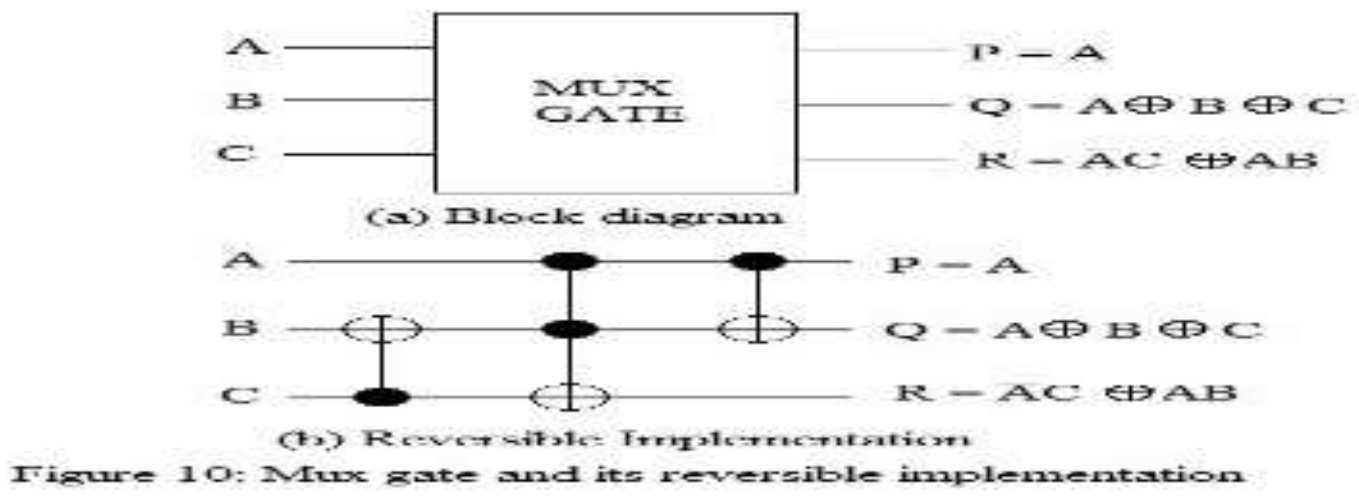

\section{Reversible Flip Flop Components}

A flip flop is a clocked sequential circuit. It is capable of storing binary information and it is affected only with the change of clock pulse. A flip flop has two outputs, one in normal binary form and the other one is its complement. We have considered only reversible flip flops that are synthesized using reversible logic. In the following section, we make a survey on RS-Flip flop, JK- Flip flop, D- Flip flop, T- Flip flop and Master-Slave Flip flop.

5.1 RS- Flip flop: RS-Flip flop is a three input flip flop. The characteristic equation is derived as bellow:

$$
\mathrm{Q}_{\mathrm{T}+1}=\overline{\mathrm{C}}_{\mathrm{P}} \mathrm{Q}_{\mathrm{t}}+\mathrm{C}_{\mathrm{p}} \mathrm{S}+\mathrm{Q}_{\mathrm{r}} \overline{\mathrm{R}} \text { and } \mathrm{SR}=0
$$

The $\mathrm{SR}=0$ specifies that both $\mathrm{S}, \mathrm{R}$ cannot equal to 1 simultaneously. For this input combination, the intermediate condition occurs. During the survey, we observe that in most cases all the irreversible gates are replaced by different reversible gate to design RS- Flip flop. Table 1 shows the comparison among the existing reversible RS-Flip flop in terms of various optimization parameters.

Table1. Comparison among existing RS-Flip flop

\begin{tabular}{|c|c|c|c|c|c|c|c|}
\hline \multicolumn{8}{|c|}{ Name of the circuits } \\
\hline NG & TG & QC & Del & CI & GO & GL & HC \\
\hline \multicolumn{8}{|c|}{ RS-Latch without complemented output using Feynman \& Peres gate [13] } \\
\hline 4 & 2 & 7 & 7 & 1 & 2 & 4 & $5 \alpha+\beta$ \\
\hline \multicolumn{8}{|c|}{ RS-Latch without complemented output using Feynman \& Toffoli gate [13] } \\
\hline 4 & 2 & 8 & 8 & 1 & 3 & 4 & $4 \alpha+\beta$ \\
\hline \multicolumn{8}{|c|}{ RS-Latch using Peres gate [13] } \\
\hline 2 & 1 & 8 & 8 & 2 & 2 & 2 & $4 \alpha+2 \beta$ \\
\hline \multicolumn{8}{|c|}{ RS-Latch using URG gate [5] } \\
\hline 2 & 1 & 14 & 14 & 2 & 2 & 2 & $8 \alpha+4 \beta$ \\
\hline \multicolumn{8}{|c|}{ RS-Flip flop using PG gate [9] } \\
\hline 4 & 1 & 16 & 12 & 4 & 5 & 3 & $8 \alpha+4 \beta$ \\
\hline \multicolumn{8}{|c|}{ RS- Flip flop using BME \& Peres gate [11] } \\
\hline 3 & 2 & 23 & 23 & 3 & 4 & 3 & $8 \alpha+5 \beta+\gamma$ \\
\hline \multicolumn{8}{|c|}{ RS-Flip flop using Fredkin \& Feynman gate [18] } \\
\hline 6 & 2 & 30 & 23 & 6 & 8 & 3 & $10 \alpha+16 \beta+8 \gamma$ \\
\hline \multicolumn{8}{|c|}{ RS-Flip flop using NG, Fredkin \& Feynman gate [10] } \\
\hline 6 & 3 & 38 & 38 & 6 & 8 & 3 & $10 \alpha+12 \beta+10 \gamma$ \\
\hline
\end{tabular}


5.2 D-Flip flop: D- Flip flop is a two input flip flop. The characteristic equation is derived as bellow:

$$
\mathrm{Q}_{t+1}=\overline{\mathrm{C}}_{\mathrm{p}} \mathrm{Q}_{\mathrm{t}}+\mathrm{C}_{\mathrm{p}} \mathrm{D}
$$

It has the ability to hold the data into its internal storage. The clock pulse $\left(\mathrm{C}_{\mathrm{p}}\right)$ is used to transfer the data from input to output. During the survey, we observe that in most cases proposed a new reversible gate and realized by it. Their main objective is to optimize the cost of circuit. Table 2 shows the comparison among the existing reversible D-Flip flop in terms of various optimization parameters.

Table2. Comparison among existing D-Flip flop

\begin{tabular}{|c|c|c|c|c|c|c|c|}
\hline \multicolumn{7}{|c|}{ Name of the circuits } \\
\hline NG & TG & QC & Del & CI & GO & GL & HC \\
\hline \multicolumn{7}{|c|}{ D-Latch without complemented output using Fredkin \& Feynman gate [4][13] } & $3 \alpha+4 \beta+2 \gamma$ \\
\hline 2 & 2 & 8 & 8 & 1 & 1 & 2 & $5 \alpha+6 \beta+4 \gamma$ \\
\hline \multicolumn{7}{|c|}{ D-Flip flop using MG-1 gate [8] } \\
\hline 1 & 1 & 8 & 8 & 1 & 1 & 1 & $4 \alpha+4 \beta+2 \gamma$ \\
\hline \multicolumn{7}{|c|}{ D Flip flop using Fredkin and Feynman gate [9] } \\
\hline 3 & 2 & 9 & 9 & 2 & 2 & 3 & $5 \alpha+6 \beta+4 \gamma$ \\
\hline \multicolumn{7}{|c|}{ D-Flip flop using SG \& Feynman gate [6] } \\
\hline 2 & 2 & 10 & 10 & 2 & 2 & 2 & \\
\hline \multicolumn{7}{|c|}{ D-Flip flop using VB-1 \& Feynman gate [12] } \\
\hline 2 & 2 & 12 & 12 & 2 & 3 & 2 & $5 \alpha+5 \beta+4 \gamma$ \\
\hline \multicolumn{7}{|c|}{ D-Flip flop using BME \& Peres gate [11] } \\
\hline 3 & 2 & 23 & 23 & 3 & 4 & 3 & $8 \alpha+5 \beta+\gamma$ \\
\hline 7 & 2 & 31 & 24 & 7 & 8 & 4 & $11 \alpha+16 \beta+8 \gamma$ \\
\hline 7 & 2 & D-Flip flop using NG, Fredkin \& Feynman gate [10] \\
\hline
\end{tabular}

5.3 JK-Flip flop: JK-Flip flop is a three input flip flop. It is a refinement of RS-Flop flop that remove the intermediate state. The characteristic equation is derived as bellow:

$$
\mathrm{Q}_{1+1}=\overline{\mathrm{C}}_{p} \mathrm{Q}_{1}+\mathrm{Q}_{1} \overline{\mathrm{K}}+\mathrm{C}_{p} \overline{\mathrm{Q}}_{\mathrm{I}} \mathrm{J}
$$

When both input ( $\mathrm{J}$ and $\mathrm{K}$ ) are 1 and the clock pulse remain enable then the output will be complement or toggle until the clock pulse goes back to disable. During the survey, we observe that in most cases proposed a new reversible gate and realized by it. Their main objective is to optimize the cost of circuit. Table 3 shows the comparison among the existing reversible JK-Flip flop in terms of various optimization parameters.

Table3. Comparison among existing JK-Flip flop

\begin{tabular}{|c|c|c|c|c|c|c|c|}
\hline \multicolumn{7}{|c|}{ Name of the circuits } \\
\hline NG & TG & QC & Del & CI & GO & GL & HC \\
\hline \multicolumn{7}{|c|}{ JK-Latch using Fredkin \& Feynman gate [13] } \\
\hline 5 & 3 & 16 & 16 & 2 & 3 & 5 & $6 \alpha+8 \beta+5 \gamma$ \\
\hline \multicolumn{7}{|c|}{ JK-Latch without complemented output using Toffoli \& Feynman gate [4] } \\
\hline 4 & 3 & 32 & 32 & 1 & 2 & 4 & $8 \alpha+7 \beta$ \\
\hline \multicolumn{7}{|c|}{ JK-Flip flop using MG-1 \& MG-2 gate [8] } \\
\hline
\end{tabular}




\begin{tabular}{|c|c|c|c|c|c|c|c|}
\hline 2 & 2 & 15 & 15 & 2 & 2 & 2 & $8 \alpha+10 \beta+7 \gamma$ \\
\hline \multicolumn{8}{|c|}{ JK-Flip flop using Fredkin \& Feynman gate [9] } \\
\hline 4 & 2 & 16 & 16 & 2 & 2 & 4 & $6 \alpha+8 \beta+4 \gamma$ \\
\hline \multicolumn{8}{|c|}{ JK-Flip flop using SG, Fredkin \& Feynman gate [6] } \\
\hline 3 & 3 & 17 & 17 & 2 & 3 & 3 & $7 \alpha+10 \beta+6 \gamma$ \\
\hline \multicolumn{8}{|c|}{ JK-Flip flop without complemented output using VB-1 \& VB-2 gate [12] } \\
\hline 2 & 2 & 19 & 19 & 2 & 3 & 2 & $7 \alpha+9 \beta+8 \gamma$ \\
\hline \multicolumn{8}{|c|}{ JK-Flip flop using Fredkin \& Feynman gate [10] } \\
\hline 10 & 3 & 54 & 54 & 10 & 12 & 5 & $16 \alpha+20 \beta+14 \gamma$ \\
\hline
\end{tabular}

5.4 T-Flip flop: T-Flip flop is a two input flip flop. The characteristic equation is derived as bellow:

$$
\mathrm{Q}_{\mathrm{t}+1}=\overline{\mathrm{C}}_{\mathrm{T}} \mathrm{Q}_{\mathrm{t}}+\mathrm{Q}_{\mathrm{t}} \mathrm{T}+\mathrm{C}_{\mathrm{p}} \overline{\mathrm{Q}}_{\mathrm{t}} \mathrm{T}
$$

It has the ability to toggle its output. During the survey, we observe that in most cases proposed a new reversible gate and realized by it. Their main objective is to optimize the cost of circuit. Table 4 shows the comparison among the existing reversible JK-Flip flop in terms of various optimization parameters.

Table4. Comparison among existing T-Flip flop

\begin{tabular}{|c|c|c|c|c|c|c|c|}
\hline \multicolumn{8}{|c|}{ Name of the circuits } \\
\hline NG & TG & QC & Del & CI & GO & GL & HC \\
\hline \multicolumn{8}{|c|}{ T-Latch without complemented output using Toffoli \& Feynman gate [4] } \\
\hline 2 & 2 & 6 & 6 & 1 & 1 & 2 & $2 \alpha+\beta$ \\
\hline \multicolumn{8}{|c|}{ Clocked T-Flip flop using Peres gate \& Feynman gate [7][36] } \\
\hline 2 & 2 & 5 & 5 & 1 & 1 & 2 & $3 \alpha+\beta$ \\
\hline \multicolumn{8}{|c|}{ T-Flip flop using Peres gate \& Feynman gate [9][13][36] } \\
\hline 3 & 2 & 6 & 6 & 2 & 2 & 3 & $4 \alpha+\beta$ \\
\hline \multicolumn{8}{|c|}{ Clocked T-Flip flop using Mux \& Double Feynman gate [19][36] } \\
\hline 2 & 2 & 7 & 6 & 1 & 2 & 2 & $5 \alpha+2 \beta+\gamma$ \\
\hline \multicolumn{8}{|c|}{ T-Flip flop without complemented output using Fredkin \& Feynman [5][36] } \\
\hline 3 & 2 & 9 & 9 & 1 & 2 & 3 & $4 \alpha+4 \beta+2 \gamma$ \\
\hline \multicolumn{8}{|c|}{ T-Flip flop using VB-1 \& Feynman gate [12] } \\
\hline 3 & 2 & 13 & 13 & 2 & 2 & 3 & $6 \alpha+5 \beta+4 \gamma$ \\
\hline \multicolumn{8}{|c|}{ Clocked T-Flip flop using Fredkin \& Feynman gate [16] } \\
\hline 5 & 2 & 17 & 17 & 2 & 3 & 5 & $7 \alpha+8 \beta+4 \gamma$ \\
\hline \multicolumn{8}{|c|}{ Clocked T-Flip flop using SG \& Feynman gate [14] } \\
\hline 3 & 2 & 19 & 19 & 2 & 3 & 3 & $11 \alpha+8 \beta+6 \gamma$ \\
\hline \multicolumn{8}{|c|}{ T-Flip flop using NG, Fredkin \& Feynman gate [10] } \\
\hline 10 & 3 & 54 & 54 & 10 & 12 & 5 & $16 \alpha+20 \beta+14 \gamma$ \\
\hline
\end{tabular}


5.5 Master-Slave Flip flop: A Master-Slave flip flop is the combination of two separate flip flops. One acts as a master and other as a slave. During the survey, we observe that in most cases it is realized by proposed reversible flip flop. Their main objective is to optimize the cost of circuit. Table 5 shows the comparison among the existing reversible Master-Slave flip flop in terms of various optimization parameters.

Table5. Comparison among existing Master-Slave Flip flop

\begin{tabular}{|c|c|c|c|c|c|c|c|}
\hline \multicolumn{8}{|c|}{ Name of the circuits } \\
\hline NG & TG & QC & Del & CI & GO & GL & HC \\
\hline \multicolumn{8}{|c|}{$\begin{array}{l}\text { Master Slave RS-Flip flop without complemented output using Fredkin, Toffoli \& Feynman gate } \\
\text { [13] }\end{array}$} \\
\hline 6 & 3 & 16 & 16 & 2 & 3 & 6 & $7 \alpha+5 \beta+2 \gamma$ \\
\hline \multicolumn{8}{|c|}{ Master Slave D-Flip flop without complemented output using Fredkin \& Feynman gate [13] } \\
\hline 4 & 2 & 16 & 16 & 2 & 2 & 4 & $6 \alpha+8 \beta+2 \gamma$ \\
\hline \multicolumn{8}{|c|}{ Master Slave D-Flip flop using MG-1 gate [8] } \\
\hline 2 & 1 & 16 & 16 & 2 & 2 & 2 & $10 \alpha+12 \beta+8 \gamma$ \\
\hline \multicolumn{8}{|c|}{ Master Slave D-Flip flop without complemented output [4] } \\
\hline 5 & 3 & 16 & 16 & 2 & 2 & 5 & $6 \alpha+8 \beta+5 \gamma$ \\
\hline \multicolumn{8}{|c|}{ Master Slave D-Flip flop using VB-1 \& Feynman gate [12] } \\
\hline 3 & 2 & 23 & 23 & 3 & 2 & 3 & $9 \alpha+10 \beta+8 \gamma$ \\
\hline \multicolumn{8}{|c|}{ Master Slave JK-Flip flop using MG-1 \& MG-2 gate [8] } \\
\hline 3 & 2 & 23 & 23 & 3 & 3 & 3 & $13 \alpha+16 \beta+11 \gamma$ \\
\hline \multicolumn{8}{|c|}{ Master Slave JK-Flip flop without complemented output using Fredkin \& Feynman gate [13] } \\
\hline 6 & 3 & 23 & 23 & 2 & 3 & 6 & $8 \alpha+12 \beta+7 \gamma$ \\
\hline \multicolumn{8}{|c|}{ Master Slave JK-Flip flop without complemented output using VB-1 \& VB-2 [12] } \\
\hline 3 & 2 & 30 & 30 & 3 & 3 & 3 & $11 \alpha+14 \beta+12 \gamma$ \\
\hline \multicolumn{8}{|c|}{ Master Slave JK-Flip flop without complemented output [4] } \\
\hline 7 & 5 & 40 & 40 & 2 & 3 & 6 & $11 \alpha+11 \beta+3 \gamma$ \\
\hline \multicolumn{8}{|c|}{ Master Slave JK-Flip flop using NG, Fredkin, Feynman gate [10] } \\
\hline 18 & 3 & 110 & 110 & 18 & 21 & 8 & $28 \alpha+24 \beta+28 \gamma$ \\
\hline \multicolumn{8}{|c|}{ Master Slave T-Flip flop without complemented output using Peres, MPG, Feynman gate [7] } \\
\hline 4 & 3 & 10 & 10 & 2 & 3 & 4 & $6 \alpha+2 \beta+\gamma$ \\
\hline \multicolumn{8}{|c|}{ Master Slave T-Flip flop without complemented output using Peres , Feynman \& Fredkin gate } \\
\hline 4 & 3 & 13 & 13 & 2 & 0 & 4 & $6 \alpha+5 \beta+2 \gamma$ \\
\hline \multicolumn{8}{|c|}{ Master Slave T-Flip flop without complemented output [4] } \\
\hline 5 & 4 & 14 & 14 & 2 & 2 & 5 & $5 \alpha+5 \beta+3 \gamma$ \\
\hline \multicolumn{8}{|c|}{ Master Slave T-Flip flop using VB-1 \& Feynman gate [12] } \\
\hline 4 & 2 & 24 & 24 & 3 & 1 & 4 & $10 \alpha+10 \beta+8 \gamma$ \\
\hline
\end{tabular}

\section{Reversible Flip Flop Components}

6.1 Reversible Counter: Counter is sequential circuit that goes through a prescribed sequence of states upon the application of input pulse. A n-bit reversible counter [37] consists of $n$ reversible flip flops. Counter is categorized into two types: Synchronous and Asynchronous. In synchronous counter, the clock pulse is applied as input of flip flops. In asynchronous counter the output of one flip flop is 2 
applied to the clock pulse of other flip flop respectively except the first flip flop. The clock pulse is applied directly to the clock pulse of the first flip flop. During the survey, we observed that it is realized by proposed flip flop and optimize the cost of circuit. Here we calculate the values of optimization parameters which is not mentioned in the respective paper. Table 6 shows the comparison among the existing reversible counter in terms of various optimization parameters.

Table6. Comparison among existing Reversible Counter

\begin{tabular}{|c|c|c|c|c|c|c|c|}
\hline \multicolumn{8}{|c|}{ Name of the circuits } \\
\hline NG & TG & QC & Del & CI & GO & GL & HC \\
\hline \multicolumn{8}{|c|}{ 4-bit Synchronous counter [22] } \\
\hline 12 & 3 & 32 & 32 & 8 & 5 & 12 & $17 \alpha+7 \beta$ \\
\hline \multicolumn{8}{|c|}{ 4-bit Synchronous counter [7] } \\
\hline 12 & 3 & 36 & 36 & 8 & 6 & 12 & $18 \alpha+12 \beta+4 \gamma$ \\
\hline \multicolumn{8}{|c|}{ 4-bit Synchronous up-down counter [19] } \\
\hline 10 & 4 & 41 & 37 & 7 & 9 & 10 & $29 \alpha+10 \beta+4 \gamma$ \\
\hline \multicolumn{8}{|c|}{ 4-bit Synchronous down counter [14] } \\
\hline 7 & 3 & 100 & 100 & 13 & 16 & 7 & $50 \alpha+37 \beta+24 \gamma$ \\
\hline \multicolumn{8}{|c|}{ 4-bit Synchronous up-down counter [14] } \\
\hline 10 & 3 & 127 & 117 & 18 & 20 & 10 & $56 \alpha+50 \beta+30 \gamma$ \\
\hline \multicolumn{8}{|c|}{ 4-bit asynchronous counter [22] } \\
\hline 8 & 3 & 23 & 23 & 4 & 1 & 8 & $9 \alpha+4 \beta$ \\
\hline \multicolumn{8}{|c|}{ 4-bit asynchronous counter [7] } \\
\hline 8 & 3 & 23 & 23 & 7 & 5 & 8 & $15 \alpha+4 \beta$ \\
\hline \multicolumn{8}{|c|}{ 4-bit asynchronous counter [20] } \\
\hline 12 & 3 & 36 & 36 & 8 & 6 & 12 & $18 \alpha+12 \beta+4 \gamma$ \\
\hline \multicolumn{8}{|c|}{ 4-bit asynchronous counter [21] } \\
\hline 23 & 2 & 71 & 71 & 15 & 12 & 12 & $31 \alpha+4 \beta+2 \gamma$ \\
\hline \multicolumn{8}{|c|}{ 4-bit asynchronous up-down counter [16][36] } \\
\hline 7 & 2 & 71 & 71 & 8 & 16 & 7 & $31 \alpha+32 \beta+16 \gamma$ \\
\hline \multicolumn{8}{|c|}{ 4-bit asynchronous up-down counter [17][36] } \\
\hline 8 & 2 & 72 & 72 & 8 & 13 & 8 & $32 \alpha+32 \beta+16 \gamma$ \\
\hline \multicolumn{8}{|c|}{ 4-bit asynchronous up-down counter [15][36] } \\
\hline 7 & 2 & 79 & 79 & 8 & 16 & 7 & $47 \alpha+32 \beta+24 \gamma$ \\
\hline \multicolumn{8}{|c|}{ 4-bit asynchronous up counter [18] } \\
\hline 28 & 2 & 124 & 92 & 28 & 32 & 16 & $44 \alpha+64 \beta+32 \gamma$ \\
\hline
\end{tabular}

6.2 Reversible Register: A register is suitable for holding binary information. An n-bit register have a group of $\mathrm{n}$ flip flops. It is referred as parallel load that means all the bits of the register are loaded simultaneously with a single clock pulse. Shift register is capable of shifting its binary information either to the right or left. All modes of operation of a universal shift register such as SISO (serial-in-serial output), SIPO (serial-in-parallel output), PISO (parallel-in-serial output) and PIPO (parallel-in-parallel output) can also be performed upon the occurrence of clock. Table 7 shows the comparison among the existing reversible universal shift register in terms of various optimization parameters. 
Table 7. Comparison among exiting Reversible Universal Shift Register

\begin{tabular}{|c|c|c|c|c|c|c|c|}
\hline \multicolumn{8}{|c|}{ Name of the circuits } \\
\hline NG & TG & QC & Del & CI & GO & GL & HC \\
\hline \multicolumn{8}{|c|}{ Universal Shift Register [30] } \\
\hline 26 & - & 94 & - & 14 & 19 & - & - \\
\hline \multicolumn{8}{|c|}{ Universal Shift Register using FF-I\& Mux-II [27] } \\
\hline 34 & - & 98 & - & 18 & 21 & - & - \\
\hline \multicolumn{8}{|c|}{ Universal Shift Register using FF-I\& Mux-I [27] } \\
\hline 41 & - & 108 & - & 28 & 33 & - & - \\
\hline \multicolumn{8}{|c|}{ Universal Shift Register [26] } \\
\hline- & - & 110 & - & - & 36 & - & - \\
\hline \multicolumn{8}{|c|}{ Universal Shift Register using FF-III\& Mux-I [27] } \\
\hline 40 & - & 110 & - & 30 & 37 & - & - \\
\hline \multicolumn{8}{|c|}{ Universal Shift Register using FF-III \& Mux-II [27] } \\
\hline 33 & - & 110 & - & 20 & 25 & - & - \\
\hline \multicolumn{8}{|c|}{ Universal Shift Register [25] } \\
\hline- & - & 114 & - & - & 40 & - & - \\
\hline \multicolumn{8}{|c|}{ Universal Shift Register [28] } \\
\hline- & - & 114 & 114 & - & 40 & - & - \\
\hline \multicolumn{8}{|c|}{ Universal Shift Register [24] } \\
\hline- & - & 120 & - & - & 36 & - & - \\
\hline \multicolumn{8}{|c|}{ Universal Shift Register using FF-II \& Mux-II [27] } \\
\hline 41 & - & 120 & - & 24 & 29 & - & - \\
\hline \multicolumn{8}{|c|}{ Universal Shift Register using FF-II \& Mux-I [27] } \\
\hline 48 & - & 130 & - & 34 & 41 & - & - \\
\hline \multicolumn{8}{|c|}{ Universal Shift Register [23] } \\
\hline- & - & 144 & - & - & 40 & - & - \\
\hline \multicolumn{8}{|c|}{ Universal Shift Register [29] } \\
\hline 48 & - & 144 & - & 24 & 31 & - & - \\
\hline
\end{tabular}

6.3 Reversible RAM: The $2^{\mathrm{n}} \mathrm{x}$ m memory consists $2^{\mathrm{n}}$ words with $\mathrm{n}$ bit address line and $\mathrm{m}$ bit in each words. It has total $\left(2^{n} . m\right)$ numbers of binary cells. Each binary cell contains one flip flop, three 3input AND gate and two NOT gate. The $\mathrm{n}$ bit address goes through $\mathrm{n} \times 2^{\mathrm{n}}$ decoder. During the survey, we observe that the construction of reversible RAM is the realization of all the irreversible components by reversible components. Their main focus is to reduce the power consumption/dissipation, quantum cost, number of garbage output and gate count. Table 8 shows the comparison among the existing reversible RAM.

Table 8. Comparison among exiting Reversible RAM

\begin{tabular}{|c|c|c|c|}
\hline \multicolumn{4}{|c|}{ Name of the circuits } \\
\hline NG & QC & GO & PC/PD* \\
\hline \multicolumn{4}{|c|}{$2^{\mathrm{n}}$ x m PRAM [31] } \\
\hline 696 & 120 & 1243 & $112 \mathrm{mV}$ \\
\hline \multicolumn{4}{|c|}{$2^{\mathrm{n}} \mathrm{x}$ m Reversible RAM [32] } \\
\hline $2^{\mathrm{n}}(8 \mathrm{~m}+2)-1$ & $58 m+4.2^{n}-7$ & $2^{n}(7 m+3)+n$ & - \\
\hline \multicolumn{4}{|c|}{$2 \times 4$ RAM [33] } \\
\hline 30 & - & 9 & $29 \mathrm{~mW}$ \\
\hline \multicolumn{4}{|c|}{$2^{\mathrm{n}} \times \mathrm{m}$ Reversible RAM [34] } \\
\hline $2^{\mathrm{n}}(6 \mathrm{~m}+2)+\mathrm{m}-1$ & $2^{\mathrm{n}}(19 m+9)-7$ & $\mathrm{~m} \cdot\left(4 \cdot 2^{\mathrm{n}}-1\right)+\mathrm{n}$ & - \\
\hline \multicolumn{4}{|c|}{$2^{\mathrm{n}} \times \mathrm{m}$ PPRRAM [35] } \\
\hline $2^{n-1}(9 m+5)+m-1$ & - & $2^{n}(4 m+4)+m \cdot n$ & - \\
\hline
\end{tabular}

$* \mathrm{PC} / \mathrm{PD}=$ Power Consumption / Power Dissipation. 


\section{Conclusion}

Designing optimized sequential reversible circuit is always a challenging task. In this paper we compute the respective optimization parameters which are not present in the existing available literature. At the same time a comparative table has been shown for better understanding of the state of art work. In this way the paper helps researchers to understand the current state of work and helps them to focus on the more optimized design and efficient reversible circuit.

\section{REFERENCES}

[01] Michael P. Frank, "Reversibility for Efficient Computing.”, PhD thesis, MIT Artificial Intelligence Laboratory, 1999.

[02] Rolf Landauer, "Irreversibility and Heat Generation in the Computing Process", IBM Journal of Research and Development, vol. 5, pp. 183-191, 1961.

[03] Charles H.Bennett, "Logical Reversibility of computation", IBM Journal of Research and Development, vol. 17, no. 6, pp. 525-532, 1973.

[04] M. L. Chuang and C.Y. Wang, "Synthesis of reversible sequential elements", ACM journal of Engineering Technologies in Computing Systems (JETC), vol. 3, no. 4, 2008.

[05] SKS Hari, S Shroff, Sk Noor Mahammad and V. Kamakoti, "Efficient Building Blocks for Reversible Sequential Circuit design”, Proceedings of the International Midwest Symposium on Circuits and Systems, 2006.

[06] Abu Sadat Md. Sayem and Masashi Ueda, “Optimization of Reversible Sequential Circuits”, Journal of Computing, vol.2, issue 6, pp. 208-214, 2010.

[07] Md. Selim Al. Mamun, B.K. Karmakar, "Design of reversible counter", International journal of advanced computer science and applications, Vol. 5, No. 1, 2014-04-03.

[08] Md. Selim Al Mamun, Indrani Mandal and Md. Hasanuzzaman, "Efficient Design of Reversible Sequential Circuit", IOSR Journal of Computer Engineering (IOSRJCE), ISSN: 2278-0661, ISBN: 2278-8727 Volume 5, Issue 6 (Sep-Oct.2012), PP 42-47.

[09] H Thapliyal and N Ranganathan, "Design of Reversible Latches Optimized for Quantum Cost, Delay and Garbage Outputs", Proceedings of Twenty Third International Conferences on VLSI Design, pp. 235- 240(2010).

[10] H. Thapliyal, M. B. Srinivas, and M. Zwolinski, "A beginning in the reversible logic synthesis of sequential circuits", In Proc. the Military and Aerospace Programmable Logic Devices Intl. Conf., Washington, Sept. 2005.

[11] Md. Belayet Ali, Md. Mosharof Hossin and Enayat Ullah, "Design of Reversible Sequential Circuit Using Reversible Logic Synthesis", International Journal of VLSI design \& Communication Systems (VLSICS) vol.2, No.4, December 2011.

[12] Bhagyalaksmi H R and Venkatesha M K, "Design of Sequential Circuit Elements Using Reversible Logic Gates", World Applied Programming, vol (2), Issue (5), May 2012. 263-271.

[13] H.Thapliyal and N. Ranganathan, "Design of reversible sequential circuits optimizing quantum cost, delay and garbage outputs", ACM Journal of Emerging Technologies in Computing Systems, vol. 6, no.4,Article 14, pp. 14:1 -14:35, Dec. 2010.

[14] “A Novel Analysis of Sequential Circuits Design Using Reversible Logic", International Journal of Research in Embedded Systems Applications- IJRAE Vol 01, Issue 02; April 2012.

[15] Prashant .R.Yelekar and Prof. Sujata S. Chiwande, "Introduction to Reversible Logic Gates \& its Application", 2nd National Conference on Information and Communication Technology (NCICT) 2011 Proceedings published in International Journal of Computer Applications ${ }^{\circledR}$ (IJCA).

[16] Mr Harish k and Mrs. Chinmaye R, "Design and Optimization of Asynchronous counter using Reversible Logic", International Journal of Engineering Research \& Technology (IJERT) ISSN: 2278-0181Vol. 4 Issue 06, June-2015.

[17] Gopalakrishnan M. and C. Karthick, “Design and Performance Analysis of Asynchronous Counter using Feynman Gate based T Flip Flop", ARPN Journal of Engineering and Applied Sciences ISSN 1819-6608 VOL. 11, NO. 17, SEPTEMBER 2016.

[18] Rohini H, Dr. Rajashekar S and Dr. PriyatamKumar, "Design of Basic Sequential Circuits using Reversible Logic", International Conference on Electrical, Electronics, and Optimization Techniques (ICEEOT) - 2016.

[19] M Tejaswini and Raji C, "Design of Optimized Synchronous Up/Down Counter Using Reversible Logic Gates", 
International Journal of Innovations in Engineering and Technology (IJIET) ISSN: 2319 - 1058 Volume 7 Issue 1 June 2016. [20] P.Lavanya, K.R.Poornima and R.Venkatesh, "Design of Synchronous cyclic code counter used by novel Reversible Gate", 2016 IJARTET.

[21] K Ramprasd , R Sudheer Kumar and G Srikanth, "A Novel Approach for Design and Verification of 4-Bit Asynchronous Counter Using Reversible Logic Gates”, International Journal of Computational Engineering Research Vol, 03 Issue, 6.

[22] Md. Mizanur Rahman, Indrani Mandal and Md. Selim Al Mamun, “An Optimized Design of Counter Using Reversible Logic", International Journal of Advanced Research in Computer and Communication Engineering Vol. 4, Issue 8, August 2015 ISSN (Online) 2278-1021 ISSN (Print) 23195940.

[23] Ashis Kumer Biswas, Lafa Jamal, M. A. Mottalib1, Ha z Md. Hasan Babu , "Design of a Reversible Parallel Loading Shift Register”, Dhaka Univ. J.Eng and Tech. vol 1(2) 1-5, 2011.

[24] D. Krishnaveni, M. Geetha Priya , "A Novel Design of Reversible Uni-versal Shift Register with Reduced Delay and Quantum Cost” Journal of Computing, Volume 4, Issue 2, February 2012, Issn2151-9617.

[25] Himanshu Thapliyal and M.B Srinivas, "A Beginning in the Reversible Logic Synthesis of Sequential Circuits” MAPLD 2005.

[26] Rashid Anwar, 2Jobbin Abraham Ben, “A Novel Design of Reversible Universal Shift Register”, IJCSMC, Vol. 3, Issue. 3, March 2014, pg.530 - 535, ISSN 2320-088X.

[27] D. Krishnaveni, M. Geetha Priya, "A Novel Design of Reversible Universal Shift Register with Reduced Delay and Quantum Cost”, Journal of Computing, Volume 4, Issue 2, February 2012, ISSN 2151-9617.

[28] Md. Selim Al Mamun, Indrani Mandal, Md. Hasanuzzaman, "Design of Universal Shift Register Using Reversible Logic", International Journal of Engineering and Technology Volume 2 No. 9, September, 2012, ISSN: 2049-3444.

[29] Thapliyal, H. and Ranganathan, N., "Design of Reversible Sequential Circuits optimizing Quantum Cost, Delay, and Garbage Outputs”, 2010 ACM J. Emerg. Technol. Comput. Syst. 6, 4, Article 14, (December 2010), 31 pages.

[30] Noor Muhammed Nayeem, Md. Adnan Hossain, Lafifa Jamal, Hafiz Md. Hasan Babu, 'Efficient Design of Shift Registers Using Reversible Logic’, 2009 International Conference on Signal Processing Systems, IEEE

[31] Shibinu A. R and Rajkumar .P, 'A Power Efficient Design of Reversible RAM Using Pseudo Reed-Muller Expression', International Journal of Science and Research (IJSR) ISSN (Online): 2319-7064

[32] Farah Sharmin, Md. Masbaul Alam Polash, Md. Shamsujjoha, Lafifa Jamal, Hafiz Md. Hasan Babu, 'Design of a Compact Reversible Random Access Memory’, 978-1-61284-836-5/11/\$26.00 @2011 IEEE

[33] S. Aathilakshmi, Mrs. R. Krishnaveni, 'Design of an Efficient Sequential NANO-RAM Circuit using Reversible Gates', IJSRD - International Journal for Scientific Research \& Development| Vol. 2, Issue 12, 2015 | ISSN (online): 2321-0613

[34] Md. Selim Al Mamun, Syed Monowar Hossain, 'Design of Reversible Random Access Memory', International Journal of Computer Applications (0975 - 8887) Volume 56- No.15, October 2012

[35] Ghahreman Pourvali, 'On Design of a Novel Nano Metric Parity Preserving Reversible Random Access Memory', International Journal of Computer Applications (0975 - 8887) Volume 75- No.4, August 2013 